\title{
Anthracene-Based Thiol-Ene Networks with Thermo-Degradable and Photo-Reversible Properties
}

Jonas Van Damme, ${ }^{\dagger, \S}$ Otto van den Berg, ${ }^{\dagger, \ddagger \S}$ Joost Brancart, ${ }^{\ddagger}$ Laetitia Vlaminck, ${ }^{\dagger}$ Carolien Huyck, ${ }^{\ddagger}$ Guy Van Assche, ${ }^{\neq}$Bruno Van Mele, ${ }^{\ddagger}$ and Filip Du Prez ${ }^{*} \oplus^{\dagger}$

${ }^{\dagger}$ Department of Organic and Macromolecular Chemistry, Polymer Chemistry Research Group, Ghent University, Krijgslaan 281, S4-bis, B-9000 Ghent, Belgium

${ }^{*}$ Research Unit of Physical Chemistry and Polymer Science (FYSC), Vrije Universiteit Brussel (VUB), Pleinlaan 2, B-1050 Brussels, Belgium

\section{Supporting Information}

ABSTRACT: Reversible networks based on an alkenefunctionalized dimer of 9-anthracenemethanol were synthesized by photoinitiated radical thiol-ene polyaddition, using either a poly(dimethylsiloxane-co-propylmercaptomethylsiloxane) or a novel aliphatic trithiol synthesized from 1,2,4trivinylcyclohexane in a simple two-step procedure. The obtained networks were analyzed using differential scanning calorimetry, dynamic mechanical analysis, polarization microscopy, X-ray diffraction, and (photo)rheology. The two types of

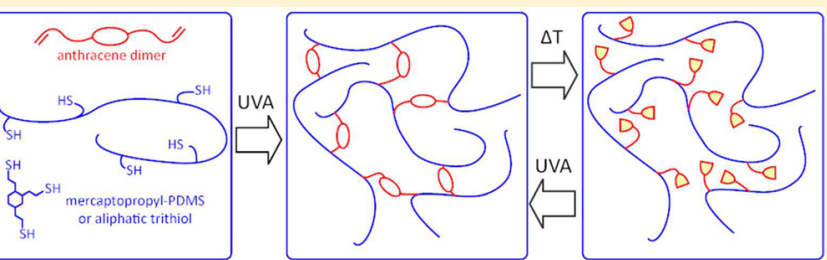

DIMER + POLYTHIOL
OIL networks showed weak endothermic transitions between 50 and $60{ }^{\circ} \mathrm{C}$, which proved to originate either from melting of a crystalline anthracene-dimer phase (trithiol network) or from a liquid crystalline phase (PDMS network) based on X-ray diffraction and polarization microscopy. Using rheology, both types of networks were shown to cleanly decompose into multifunctional anthracene monomers at temperatures above $180^{\circ} \mathrm{C}$. Irradiation of these anthracene monomers resulted in the formation of networks having similar physical properties as the original materials.

\section{INTRODUCTION}

Covalently cross-linked materials are used in numerous applications, ranging from coatings, over composite materials, to biomedical materials. The presence of covalent cross-links makes these materials behave in a predominantly elastic manner when exposed to mechanical deformation. However, the permanently cross-linked structure often poses a serious disadvantage when reshaping, recycling or easy removal of the material is required. Introduction of reversible covalent bonds into a network allows for combining the advantages of classical cross-linked materials, e.g., dimensional stability, with the advantages of non-cross-linked oligomers or thermoplastic polymers, e.g., easy processing. The type of reversible covalent bond determines the conditions under which such materials behave as classic thermosets or as thermoplastic materials. A reversible bond can either be dynamic, involving some form of exchange reaction, or nondynamic, requiring stimuli to either break or form the covalent bond. ${ }^{1}$ In addition to the type of reversible bond, the change in connectivity and the time scale on which this change of connectivity occurs largely determine the material behavior. In case associative exchanges take place, i.e., an exchange reaction where no change in macroscopic connectivity occurs, the material behaves like a strong glass former and is processable over a broad temperature range. Recently this class of materials was named "vitrimers" by Montarnal et al. as a result of the similarities in rheological behavior to a silica glass melt. ${ }^{2}$ Research on vitrimers has also been published and reviewed by our group. ${ }^{3,4}$ In the case that dissociative exchanges occur, e.g., due to a temperature dependent shift of the chemical equilibrium between a connected and an unbound state, large changes in viscosity are observed in the processing of the material as a result of large changes in connectivity.

For networks held together by nondynamic reversible bonds at the relevant time-scales, activation of the reversibility leads to a net breaking of bonds and possible conversion of an essentially solid material into a viscous liquid. As no reversion takes place when ceasing the stimulus, the connectivity of the material at ambient conditions can be tuned. Activation of the reverse reaction (with another stimulus) leads to the reformation of the network and recovery of the solid properties. Well known examples are materials containing cinnamate esters, ${ }^{5,6}$ coumarines, ${ }^{7}$ and anthracenes. ${ }^{8}$ While most of these materials dimerize upon UVA irradiation via $2 \pi-2 \pi$ photochemical cycloaddition (cinnamates and coumarines), only few occur by a $4 \pi-4 \pi$ (anthracene) photocycloaddition. Scission of the photodimers is usually accomplished by irradiation with highly energetic UVC radiation. As the $4 \pi-4 \pi$ photocycloadditions of anthracene occur using less energetic, longer wavelengths (even visible light) compared to their $2 \pi-2 \pi$

Received: November 6, 2016

Revised: February 12, 2017

Published: February 21, 2017 
counterparts, they are preferred in order to avoid side reactions. Additionally, anthracene dimers are also able to dissociate cleanly at elevated temperatures through a diradical transition state (Scheme 1). The rate of this dissociation depends on the substituents on the anthracene ring and can be brought to relevant time and temperature scales.

Scheme 1. Dimerization of Anthracene with Photochemical $4 \pi-4 \pi$ Concerted Cycloaddition and Scission of the Anthracene Dimer Back into Anthracene, either by a Thermally Activated (Radical) Reaction or by a Photochemical $4 \pi-4 \pi$ Concerted Retro Cycloaddition
2<smiles>[R]c1c2ccccc2cc2ccccc12</smiles>
$h v(>300 \mathrm{~nm})$ $h v(<300 n m)$

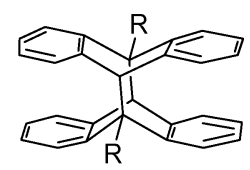

$x$
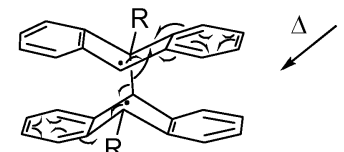

Therefore, this paper will focus on the preparation and study of nondynamic reversible anthracene dimer containing networks, where the bond-forming reaction is a concerted $4 \pi-4 \pi$ photochemical cycloaddition, and the bond-breaking reaction is a nonconcerted thermally activated retro-cycloaddition. ${ }^{10}$

This system allows the creation of cross-linked materials that are easy to repair by combining a heat treatment with UVA irradiation, either separately or simultaneously. Unlike other recent work on reversible anthracene networks, ${ }^{11}$ the (first) network formation does not occur by anthracene dimerization, but by thiol-ene reactions with anthracene dimers. This approach guarantees the synthesis of networks without free anthracene groups (and thus also no cross-linking gradient) and facilitates incorporation of the reversible bonds in known formulations and thicker materials.

A large number of publications on thiol-ene chemistry involve modification and functionalization of existing (polymer) structures and the preparation of new molecular architectures. Some excellent reviews dealing with this topic were published. ${ }^{12-14}$ In patent literature, thiol-ene polymerization of multifunctional thiols with multifunctional enes and ynes is described for the preparation of materials with different types of functionality. For example, Bowman et al. reported the polymerization of several multifunctional unsaturated urethanes, allylethers, acrylates, and methacrylates with multifunctional thiols, yielding cross-linked end products exhibiting shape memory properties, ${ }^{15}$ which are claimed for medical applications. Other examples of applications of thiol-ene based polymeric structures include the preparation of low gas permeability membranes, ${ }^{16}$ sealants, ${ }^{17}$ stamps for lithography, ${ }^{18,19}$ degradable polymeric structures for biomedical ${ }^{20}$ and dental applications, ${ }^{21-23}$ liquid crystalline compositions for optical applications, ${ }^{24}$ and polymer electrolytes for, e.g., batteries. $^{25}$

Multifunctional thiol cross-linkers can come in different shapes and forms. Silicone-based thiols have been described for different applications, ${ }^{12}$ including fast-cure optical fiber coatings, ${ }^{26}$ functionalized microfluidic devices ${ }^{27}$ and for the modification of surfaces. ${ }^{28}$ In a recent paper, we described the preparation of low-modulus dry silicone gels built on silicone-based thiols. ${ }^{29}$ A very common class of readily available tetrafunctional thiol cross-linkers is based on the esters of pentaerythrytol or trimethylolpropane and 2-mercaptoacetic acid or 3-mercaptopropanoic acid. However, the presence of multiple ester moieties in such multithiols can result in a reduced hydrolytic stability. For materials where ester moieties need to be avoided, no economically viable alternative to such tetrathiols is available. In this context, we explored the synthesis of a purely aliphatic multithiol from an available polyunsaturated precursor.

Starting from the synthesized multifunctional thiols and enefunctionalized anthracene dimers, cross-linked polymer networks containing reversible covalent bonds will be prepared that can be used either as a material that can relax mechanical stress by stimulated reshuffling of the components that make up the network or as a material that can be easily removed after its service life in applications like coatings or composite materials, by a simple temporarily temperature increase.

\section{RESULTS AND DISCUSSION}

Synthesis. Anthracene dimers substituted at the 9-position have been used as photochemically reversible linkers in a wide range of applications. ${ }^{30-32}$ On the other hand, their use as thermally labile linkers is much less explored. ${ }^{8}$ Although anthracene in its monomeric form is prone to radical addition of thiyl radicals at the 9- or 10-positions, ${ }^{33}$ the loss of $\mathrm{sp}^{2}$ hybridization of the same positions after dimerization leads to the loss of thiol-ene reactivity. Introduction of additional double bond functionality allows dimers to be used in the construction of one, two and three-dimensional structures. Since the unfunctionalized anthracene dimer itself is virtually insoluble in any solvent, functionalization of the basic anthracene dimer core is also necessary to improve and optimize the solubility of the anthracene dimer based compounds in various monomers, coreactants and solvents. In this context, an anthracene dimer derivative is used having long alkyl spacers. This derivative was made according to a reported procedure by our laboratories from 9-anthracenyl methanol and undec-10-enoyl chloride, which are both readily available and relatively low in cost (Scheme 2). ${ }^{9}$ This dimer can be applied as bifunctional building block in thiol-ene networks

Scheme 2. Preparation of Soluble, Ene-Functional Anthracene Dimers $3^{a}$

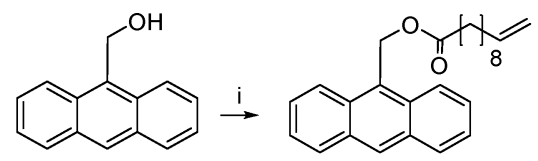

2

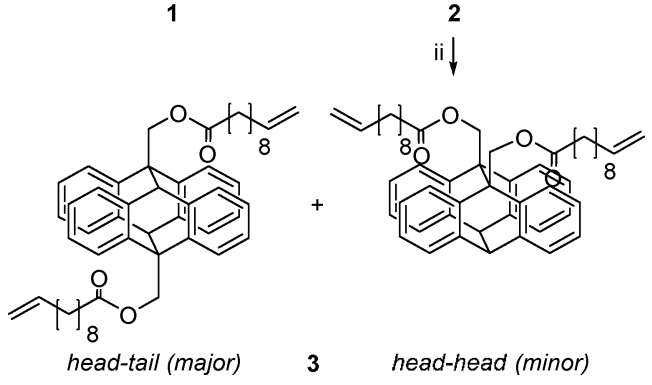

${ }^{a}$ Key: (i) undec-10-enoyl chloride, dry pyridine, $71 \%$ yield; (ii) 365 nm UV, hexane, $\geq 90 \%$ yield. ${ }^{9}$ 
where photoreversibility or thermo-/photoreversibility is required as a property of the final product, without excessive costs or a limited availability of the component.

In order to investigate the basic thermal and photochemical properties of thiol-ene networks prepared from 3, two different types of thiols were considered, i.e. one that can create well-defined anthracene-dimer based thiol-ene networks with a high concentration of anthracene-dimers and a fixed cross-link functionality of 3 , and one that results in a lower concentration anthracene-dimer network with cross-link functionalities mostly between 3 and 6 . A first cyclo-aliphatic trithiol was prepared from 1,2,4-trivinylcyclohexane (4, Scheme 3 ), a relatively inexpensive intermediate that is produced on large scale by isomerization of 1,5,9-cyclododecatriene, which is a precursor of nylon-12, over a palladium catalyst. ${ }^{34}$

Scheme 3. Preparation of a Mixture of Trifunctional Thiol Isomers $^{a}$

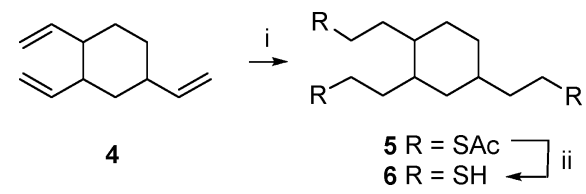

${ }^{a}$ Key: (i) thioacetic acid, DMPA, $365 \mathrm{~nm}$ UV, THF, 100\%; (ii) dilute $\mathrm{HCl}$, reflux, $90 \%$.

Since 4 is supplied as a mixture of several (stereo) isomers, reaction of $\mathbf{4}$ with thioacetic acid results in a complex mixture of trifunctional thioesters. However, a mixture of isomers is desirable in this case, since the presence of different molecular configurations suppresses crystallization, both in the thiol and in the resulting networks. Indeed, suppression of crystallization in the networks is needed to create sufficient mobility for the dimerization reaction and the thermal/photochemical scission reaction to take place. The reaction between 4 and thioacetic acid proved to be self-initiating and exothermic, requiring efficient cooling. While a conversion of up to $97 \%$ could be accomplished after an hour without initiator or irradiation and cooling using a salty ice bath, a final irradiation in the presence of a photoinitiator (2,2-dimethoxy-2-phenylacetophenone, DMPA) was performed in order to ensure (nearly) full conversion of all available double bonds to the desired end product. The proton NMR spectrum of the resulting mixture of isomers showed broad peaks with slightly overlapping $\alpha$ $\mathrm{CH}_{2} \mathrm{SAc}$ (anti-Markovnikov) and/or $\alpha$-CHSAc (Markovnikov) signals between 2.5 and 3 ppm (Figure $\mathrm{S} 1$ ). Therefore, ${ }^{13} \mathrm{C}$ and HSQC NMR spectra were taken which exclude the presence of Markovnikov products (Figures S2 and S3).

Hydrolysis of the trithioacetate mixture $\mathbf{5}$ with dilute hydrochloric acid and subsequent vacuum distillation provided the free trithiols $6\left({ }^{1} \mathrm{H}\right.$ NMR in Figure S4). GC-MS analysis of this complex mixture gave a set of unseparated peaks of identical molecular mass components $(M=264.10 \mathrm{~g} / \mathrm{mol})$, indicative of clean conversion of the trithioacetate into the free trithiol (Figure S5) with only a minor impurity of dithiol $(M=$ $230 \mathrm{~g} / \mathrm{mol})$.

As alternative thiol-bearing compound for network synthesis, a readily available thiopropyl functional PDMS $\left(M_{\mathrm{n}}=6800 \mathrm{~g} /\right.$ mol, $\sim 5$ thiols per PDMS chain) is chosen. Because of the higher molecular weight to functionality ratio, a lower weight percentage of the (more expensive and solid) dimer is required for equimolarity. Additionally, due to the higher functionality, the minimal anthracene dimer-monomer ratio required to maintain the structural integrity of the network should be lower. The low $T_{\mathrm{g}}$ of PDMS-based networks also ensures enough mobility for reversible reactions to occur within the network.

Preparation of the networks based on the anthracene diene (3) and the synthesized aliphatic trithiol (6) or the available thiopropyl functional PDMS (7) required the use of a cosolvent in order to create homogeneous mixtures (having 79 and $58 \mathrm{wt}$ $\%$ of THF) at room temperature. Higher solid contents are obtainable by using better solvents (e.g., 72 and 47 wt \% of $\mathrm{CHCl}_{3}$ ), increased temperatures, and/or appropriate reactive diluents. UV-cure occurred in the presence of DMPA between glass windows separated with $1 \mathrm{~mm}$ silicone rubber spacers. After curing and removal from the mold, the solvent was allowed to evaporate under ambient conditions, resulting in clear, rigid, trithiol-based films (A) or in clear, highly elastic thiopropyl-functional-PDMS-based films (B) (Scheme 4).

Scheme 4. Preparation of Thiol-Ene Networks Using Trithiol 6 and Thiol-Functionalized PDMS 7, Resulting in a Densely Cross-Linked Structure Containing Well-Defined Cross-Links (A) and a Loosely Cross-Linked Structure with Ill-Defined Polymeric Cross-Links $(\mathrm{B})^{a}$
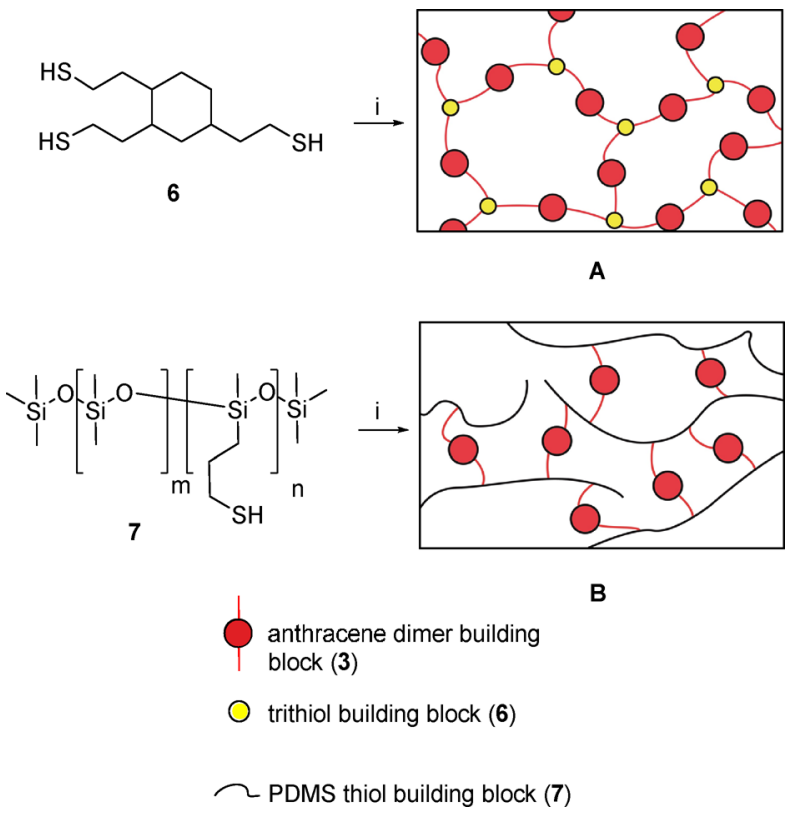

${ }^{a}$ Key: (i) anthracene dimer diene 3, DMPA, 365 nm UV (1 h), THF.

Thermal Characterization. Pure dimer 3 is characterized by a broad highly structured melting peak at $148{ }^{\circ} \mathrm{C}$ measured via differential scanning calorimetry (DSC), which might indicate recrystallization of $\mathbf{3}$ into thermally more stable crystal structures. While the melting endotherm of 3 is quite obvious, the scission of the dimer into the monomeric anthracene can only be observed as the small exothermic bending of the baseline near $200{ }^{\circ} \mathrm{C}$ in the DSC curve of pure 3 (Figure 1).

It was previously reported that heating dimer 3 for $10 \mathrm{~min}$ led to scission into its corresponding monomer 2 starting from temperatures around $160-170{ }^{\circ} \mathrm{C}$, without appreciable competing side reactions (Figure 2 and Figure 3). ${ }^{9}$ This proves that the melting endotherm of $\mathbf{3}$ and the scission into $\mathbf{2}$ do not overlap significantly on the temperature scale of the DSC 


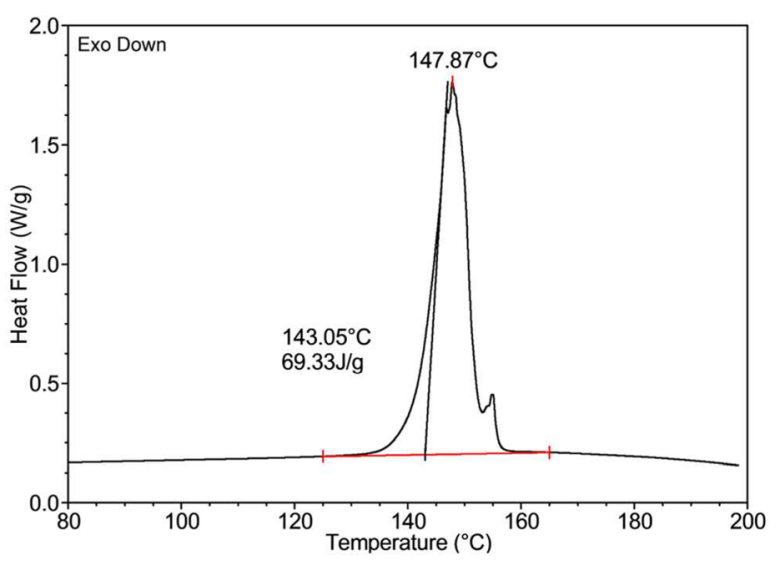

Figure 1. DSC curve of 3 (first heating) showing the melting endotherm and a lack of other visible thermal changes in the area where thermal scission of 3 into monomeric anthracene is to be expected according to NMR (Figure 3).

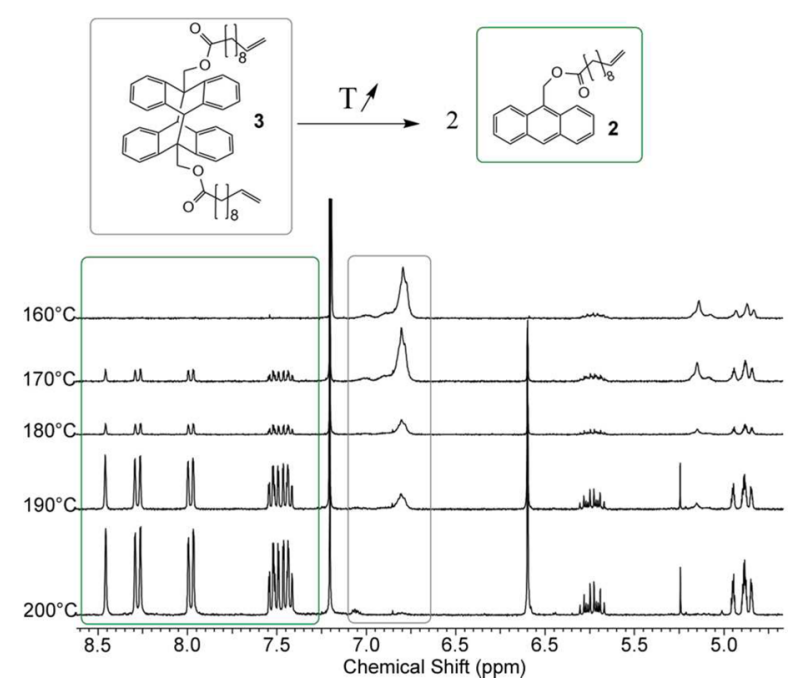

Figure 2. Proton NMR spectra of dimer 3 after 10 min of heating at the indicated temperatures, showing the disappearance of the dimer related resonance at $6.8 \mathrm{ppm}$ and the appearance of typical peaks for the 9-substituted anthracene 2 as temperature increases.

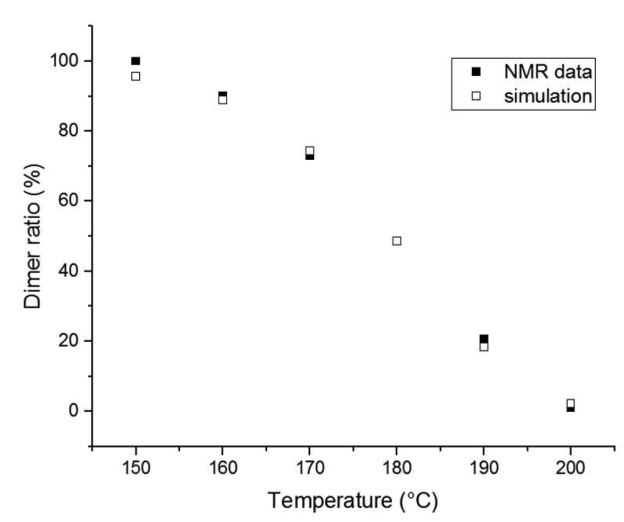

Figure 3. Percentage of remaining dimer 3 after $10 \mathrm{~min}$ of heating at the indicated temperatures, as determined by ${ }^{1} \mathrm{H}$ NMR and simulated using the derived Arrhenius parameters.

experiment and that the exothermic bending of the baseline starting from 160 to $170{ }^{\circ} \mathrm{C}$ indeed originates from the scission. Using the proton NMR data of the controlled bulk thermal dissociation of 3 , the Arrhenius parameters were calculated. The derived pre-exponential factor $\ln A$ was 32.5 while the activation energy $E_{\mathrm{a}}$ was $148 \mathrm{~kJ} / \mathrm{mol}$. These results are lower but also more reliable than those previously reported in solution $\left(\ln A=38.7\right.$ and $\left.E_{\mathrm{a}}=176 \mathrm{~kJ} / \mathrm{mol}\right)$. In Figure 3, the good resemblance between the experimental data and the performed simulations using those new data are shown. Using the new parameters, the scission conversion at the end of the DSC measurement was calculated to be $24 \%$ and a (small) peak maximum would only be reached at $210^{\circ} \mathrm{C}$. This corresponds to a connectivity $x=0.76$, where $x=1$ for fully dimerized/ cross-linked systems and $x=0$ for fully dissociated systems. This limited conversion, in combination with a (presumably) low scission enthalpy explains the low intensity in the DSC experiment.

Networks based on 3 and trithiol 6 (network A) or PDMS multithiol 7 (network B), both containing the same type of reversible bonds, were subsequently investigated. While trithiol based network $\mathbf{A}$ has a high cross-link density, PDMS based network $\mathbf{B}$ has a low one. After UV cure and evaporation of the THF, first under ambient conditions and next in high vacuum, the samples were measured using differential scanning calorimetry (DSC). The glass transition of network $\mathbf{A}$ is rather difficult to observe at $10 \mathrm{~K} / \mathrm{min}$ in the first heating, but could be measured in the second heating, being equal to $14.6{ }^{\circ} \mathrm{C}$ (Figure 4). As expected, network $\mathbf{B}$ has a very low glass

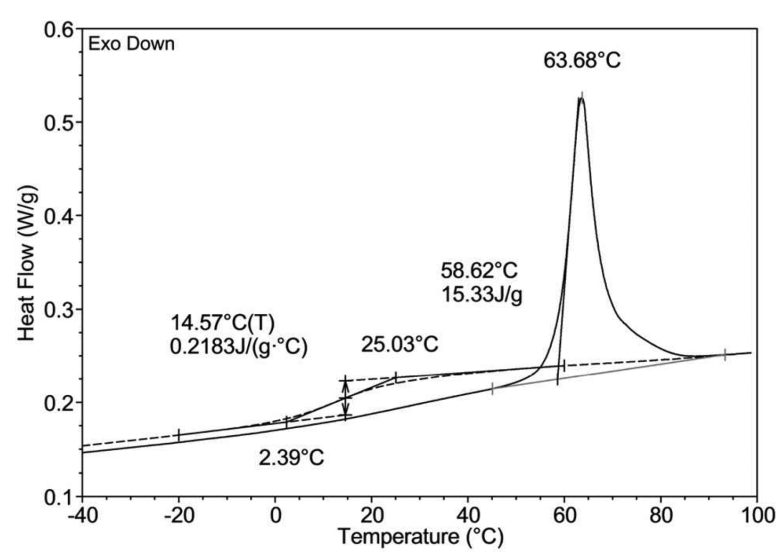

Figure 4. DSC curve of network A based on 3 and trithiol 6. First (full lines) and second (dotted lines) heating at $10 \mathrm{~K} / \mathrm{min}$.

transition temperature $\left(T_{\mathrm{g}}=-118^{\circ} \mathrm{C}\right)$ because of the flexible PDMS chains and the low cross-linking density. Both materials show a weak endothermic peak at around $50-70{ }^{\circ} \mathrm{C}$ (Figures 4 and 5). Especially for network $\mathbf{A}$, it is surprising that any crystallinity is present, as crystallinity in highly cross-linked materials is usually suppressed. However, due to the method of preparation, which involves the use of a good solvent for dissolving all reactants, the initial structure has a much higher degree of molecular mobility than usually observed for materials cross-linked in bulk. In fact, our materials can be described as typical xerogels, formed by cross-linking in concentrated solution, followed by slow air drying, which allows the components to slowly self-assemble to a thermodynamically more stable ordered situation. This is in stark contrast to densely cross-linked networks that are crosslinked in bulk, where mobility is quickly reduced by the formation of the polymer network, freezing to an amorphous material that is far away from thermodynamic equilibrium. 


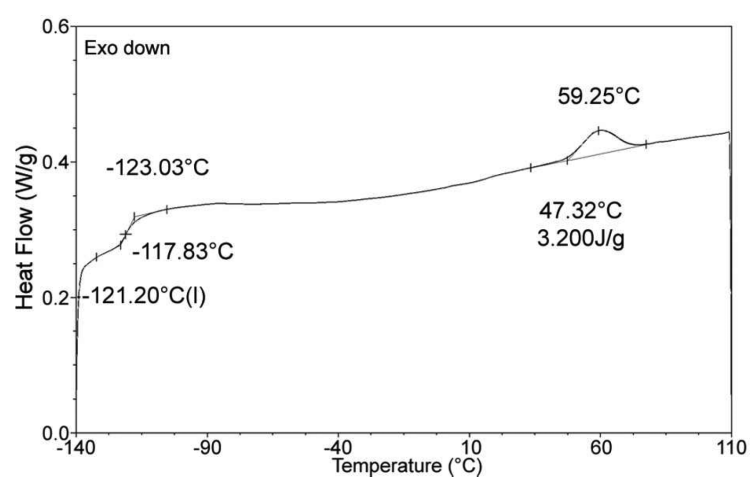

Figure 5. DSC curve of network B based on 3 and PDMS 7. First heating at $10 \mathrm{~K} / \mathrm{min}$.

Vitrification of the network in bulk will also halt the crosslinking at the point where the bulk- $T_{\mathrm{g}}$ coincides with the reaction temperature, leading to an incomplete reaction. However, even when the overall content of 3 in the networks is taken into account, the observed endotherms are rather small, respectively $16.8 \mathrm{~J} /(\mathrm{g}$ of 3 ) for network $\mathbf{A}$ and $15.8 \mathrm{~J} /(\mathrm{g}$ of 3 ) for network B, or about four times smaller than for pure 3 (Figure 1).

The reduced melting temperature of the networks $\mathbf{A}$ and $\mathbf{B}$, combined with a decreased melting enthalpy, as compared to the anthracene dimer 3, might suggest the formation of a mesophase, which could be induced by the presence of mobile spacers and/or a mobile low $T_{\mathrm{g}}$ polymer, such as the PDMS in network B, combined with a rigid anisotropic anthracene dimer moiety. In order to determine whether the prepared materials exhibit either crystalline or liquid crystalline order, both networks were characterized using wide-angle X-ray diffraction (Figures S6 and S7). Both samples were first analyzed at ambient temperature, after which they were quickly heated to just above the transition temperatures $\left(70{ }^{\circ} \mathrm{C}\right)$, quenched to room temperature, and measured again. Finally the samples were measured $48 \mathrm{~h}$ after quenching. From the first diffraction results, it was found that network A based on trithiol 6 shows sharp reflections at $2 \theta$ values of 11,18 , and 28 degrees, which correspond with $d$-spacings of $8 \AA, 4.9$ and $3.2 \AA$, respectively. While the trithiol-based network A displays sharp XRD patterns typical for a crystalline material, the PDMS-based network B shows a much more diffuse reflection at 12 degrees, corresponding with a $d$-spacing of $7.3 \AA$, which is indicative for a nematic liquid crystalline material that exhibits orientational order without positional order.

The subsequent heating and quenching step initially leads to the formation of amorphous materials at room temperature. However, after resting for $24 \mathrm{~h}$ at room temperature, the PDMS-based material regains its liquid crystalline order, while the trithiol-based material remains amorphous. Polarization microscopy of a freshly prepared sample of $\mathbf{B}$ exhibited birefringent domains, when observed between crossed polarizers, confirming that $\mathbf{B}$ is a liquid crystalline rubbery material (Figure 6).

Dynamic Mechanical Analysis and Rheology. The viscoelastic behavior of thiol-ene network $\mathbf{A}$ was characterized using DMA (Figure 7). The decrease in storage modulus and maximum in loss modulus, observed around $40{ }^{\circ} \mathrm{C}$, can be attributed to the glass transition of $\mathbf{A}$. This is in good correlation to the DSC experiment on network A, where a slope in the baseline can be seen in the first heating before the

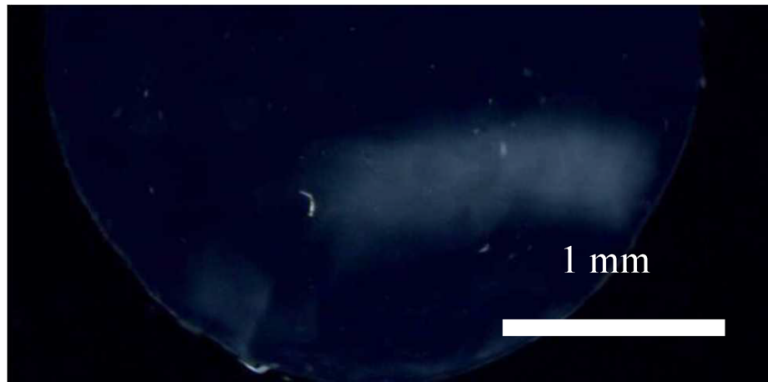

Figure 6. Optical micrograph of a thin film $(100 \mu \mathrm{m})$ of $\mathbf{B}$ recorded with crossed polarizers.

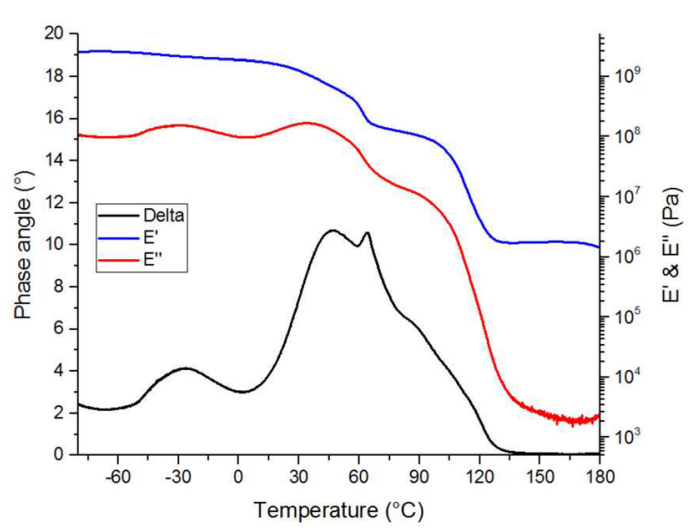

Figure 7. DMA plot of network A based on 3 and trithiol 6 , first heating at $2.5 \mathrm{~K} / \mathrm{min}$ between -80 and $+180{ }^{\circ} \mathrm{C}$, showing the storage and loss modulus as a function of temperature.

melting occurs. A further increase in temperature above the onset of melting of the crystalline fraction of $\mathrm{A}$ at $60^{\circ} \mathrm{C}$ results in a decrease in storage modulus and loss modulus to a plateau (due to the dimer aggregates) above $100 \mathrm{MPa}$ and around 10 $\mathrm{MPa}$, respectively. Near $125{ }^{\circ} \mathrm{C}$ the moduli decrease again, resulting in a material that displays an elastomeric behavior with a storage modulus of about $2 \mathrm{MPa}$.

Beyond $180{ }^{\circ} \mathrm{C}$ the storage modulus drops sharply, and the sample turns into a highly viscous liquid. The onset thereof can be seen around $165{ }^{\circ} \mathrm{C}$. This transition corresponds with the loss of cross-linking (decrease in connectivity $\mathrm{x}$ ) due to scission of the dimer units into monomeric anthracene units. The $G^{\prime}-$ $G^{\prime \prime}$ crossover, which can be regarded as the point where a material changes from a cross-linked material into a viscous liquid (degelation), could not be studied in DMA as the material liquefied too quickly. We calculated using the acquired Arrhenius parameters that $25 \%$ of the dimers have cleaved at $180{ }^{\circ} \mathrm{C}$, leading to a connectivity $x$ equal to 0.75 . This connectivity is indeed still above the minimal connectivity for a gel of 0.50 , according to the Flory-Stockmayer equation. This (de)gelation point should be reached upon continuation of the experiment to $189^{\circ} \mathrm{C}$.

The intrinsically low modulus of network $\mathbf{B}$, compared to $\mathbf{A}$, makes the material more suitable for a viscoelastic characterization by dynamic rheometry. Rheological analysis of a $200 \mu \mathrm{m}$ thick film of $\mathbf{B}$ was performed between parallel plates at frequencies between 0.1 and $10 \mathrm{~Hz}$ and temperatures from 30 ${ }^{\circ} \mathrm{C}$ up to $200{ }^{\circ} \mathrm{C}$ (Figure 8). Increasing the temperature leads to a stepwise decrease in both storage and loss moduli between 35 and $55{ }^{\circ} \mathrm{C}$, which is slightly below the isotropization point found by DSC $\left(59^{\circ} \mathrm{C}\right)$. However, the heating rate at which the 


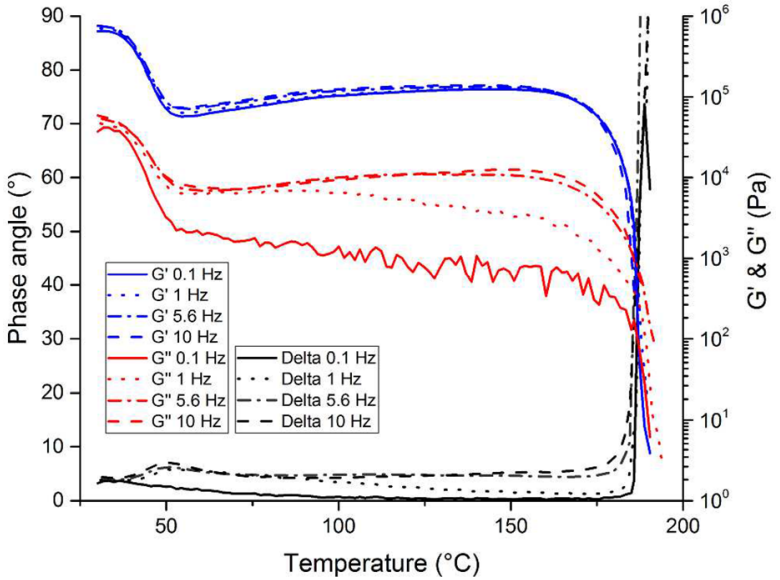

Figure 8. Dynamic rheometry curves of network B based on 3 and PDMS polythiol 7: $G^{\prime}, G^{\prime \prime}$ and phase angle as a function of temperature $(1 \mathrm{~K} / \mathrm{min}$ heating rate).

rheological measurement was performed $(1 \mathrm{~K} / \mathrm{min})$ is one decade slower than the heating rate for the DSC experiment, which explains the small difference in transition temperatures. Between 55 and $170{ }^{\circ} \mathrm{C}$ the storage modulus of $\mathbf{B}$ is slightly increasing but remains virtually constant at a low value of 0.1 $\mathrm{MPa}$, while the phase angle stays below 5 degrees; in this temperature range the material shows a typical entropy-elastic behavior. Beyond $170{ }^{\circ} \mathrm{C}$ both $G^{\prime}$ and $G^{\prime \prime}$ steeply decrease, $G^{\prime}$ faster than $G^{\prime \prime}$, until the values become equal at the point where cross-linking is lost, at around $185^{\circ} \mathrm{C}$, and the material starts to behave as a viscoelastic liquid rather than as an elastomer. Using the calculated Arrhenius parameters, a connectivity $x$ of 0.33 remains at this point in the measurement. This is higher than the minimal connectivity for a gel $(x=0.25)$, which should be reached at $187.4{ }^{\circ} \mathrm{C}$. This underestimation of the connectivity required for a gel can be explained by a significant amount of intramolecular bond formation which occurred due to the considerable dilution during cure.

The results indicate that the anthracene dimer diene 3 can effectively be used to form cross-linked materials using thiolene chemistry. By heating the cross-linked materials above 170 ${ }^{\circ} \mathrm{C}$, the dimer units split into two anthracene moieties, effectively reversing the cross-linking. The reverse process, i.e. the re-establishment of the network, should be possible by UVA-irradiation. In order to establish to what extent this reformation of the network can actually be accomplished, a 200 $\mu \mathrm{m}$ thick sample of thermally degraded $\mathbf{B}$ (prepared by heating virgin $\mathrm{B}$ for $10 \mathrm{~min}$ at $185{ }^{\circ} \mathrm{C}$ under argon), was exposed to UVA radiation in a UV rheometry setup, while measuring $G^{\prime}$, $G^{\prime \prime}$ and the phase angle at a fixed frequency of $10 \mathrm{~Hz}$ and at 60 ${ }^{\circ} \mathrm{C}$. The results are shown in Figure 9.

Irradiation clearly re-establishes the material's mechanical integrity (increase in connectivity $x$ ). The crossing of $G^{\prime}$ and $G^{\prime \prime}$ (gelation), indicating the formation of an elastic solid, occurs after $75 \mathrm{~s}$ of irradiation at approximately $2 \mathrm{~W} / \mathrm{cm}^{2}$. After $300 \mathrm{~s}$ of UV-exposure the rheological properties hardly change anymore and a solid rubber film is obtained. At $20^{\circ} \mathrm{C}$, the photochemical dimerization of dissociated $\mathbf{B}$ is much slower: the $G^{\prime}-G^{\prime \prime}$ crossover occurs after more than 20 min (Figure S8). Closer inspection reveals the most important reason for the observed differences in reactivity: dissociated $\mathbf{B}$ is an opaque (highly scattering) viscous liquid at room temperature, and becomes a clear nonscattering liquid around $50^{\circ} \mathrm{C}$, close to

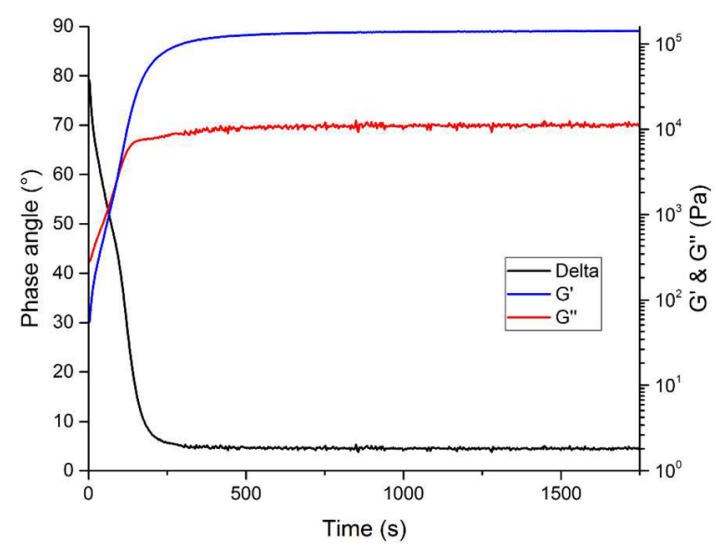

Figure 9. Dynamic rheometry curves of thermally degraded network B (200 $\mu \mathrm{m}$ thickness) during UVA irradiation $(300-400 \mathrm{~nm})$ with an intensity of approximately $2 \mathrm{~W} / \mathrm{cm}^{2}$ at $60^{\circ} \mathrm{C}: G^{\prime}, G^{\prime \prime}$, and phase angle $(10 \mathrm{~Hz})$ as a function of time.

where the isotropization occurs. This observation indicates microphase separation between the anthracene containing parts of the material and the PDMS. Such phase separation can slow down cross-linking due to the scattering of the UV light, bringing down the effective absorbed dose of UV by the sample.

For the more highly cross-linked network $\mathbf{A}$, the photorheological characterization of the network reformation upon UVA exposure at room temperature is quite challenging, as vitrification induces large changes in the modulus upon crosslinking. Besides, the reduction in chain segment mobility upon vitrification can slow down the reactions and can be a limiting factor in the degree of conversion. For this reason, the reestablishment of the network by UVA-irradiation was investigated at $60{ }^{\circ} \mathrm{C}$, above the $T_{\mathrm{g}}$ of network A. To thermally decompose network $\mathbf{A}$ into its liquid anthracene counterpart, a sample of virgin A was heated for $10 \mathrm{~min}$ at $185{ }^{\circ} \mathrm{C}$ under argon. The sample was subsequently exposed to UVA radiation at $60{ }^{\circ} \mathrm{C}$ in a UV rheometry setup, while measuring $G^{\prime}, G^{\prime \prime}$ and the phase angle at a fixed frequency of $8 \mathrm{~Hz}$. The results are shown in Figure 10.

The crossover of $G^{\prime}$ and $G^{\prime \prime}$ is found at $1200 \mathrm{~s}$ of UVA exposure, which is much later compared to the PDMS-based $\mathbf{B}$

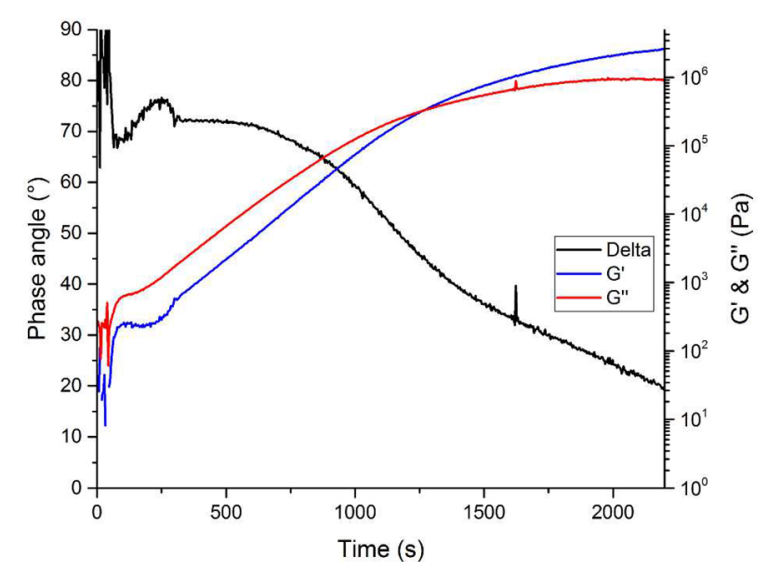

Figure 10. Dynamic rheometry curves for a film of thermally degraded A (200 $\mu$ m thickness) during UVA (300-400 nm) irradiation with an intensity of approximately $2 \mathrm{~W} / \mathrm{cm}^{2}$ at $60^{\circ} \mathrm{C}: G^{\prime}, G^{\prime \prime}$, and phase angle $(8 \mathrm{~Hz})$ versus time. 
material. The storage and loss moduli continued to increase after the crossover beyond the measurable range of the rheometer.

The difference in gelation time has several causes. First, a higher minimal connectivity is required for gelation of network A $(x=0.50$ instead of 0.25$)$. Second, the connectivity $x$ before irradiation is not 0 , and although the same anthracene chemistry is used, this initial connectivity might also differ between the materials. A third difference is the higher concentration of anthracene groups in network A. Because of this higher concentration, more energy (and thus irradiation time) is required for changes in connectivity.

The above results show that the two types of prepared thiolene networks containing anthracene dimers as reversible bonds in the interconnecting chains can be effectively split into the anthracene functionalized building blocks by heating to a temperature of $180{ }^{\circ} \mathrm{C}$ for $10 \mathrm{~min}$ under an inert atmosphere. Exposure of the resulting anthracene functionalized building blocks to UVA at slightly elevated temperature $\left(60^{\circ} \mathrm{C}\right)$ gives networks with mechanical properties that are close to the original networks. Exposure to UVA at room temperature resulted, for the two investigated types of materials, into inefficient photocuring reactions, either due to inhomogeneities, in the case of the PDMS-based network, or due to limitations to the conversion because of vitrification, in the case of the trithiol-based network.

\section{CONCLUSIONS}

A soluble alkene-functionalized dimer of 9-anthracenemethanol (3), prepared from readily available compounds, was combined with two multifunctional thiols, i.e. one well-defined mixture of isomeric trithiols (6), prepared from 1,2,4-trivinylcyclohexane in a simple two-step procedure, and an available thiopropyl functionalized PDMS (7). The networks formed from these two types of thiols resulted in a hard, cross-linked material (A; from trithiol 6) and a soft, flexible rubber (B; from PDMS thiol 7). All anthracene dimer containing materials, independent from the type of network these were included in, decomposed into anthracene functionalized building blocks in the same temperature range as the parent anthracene dimer (3), i.e., above $160{ }^{\circ} \mathrm{C}$. DSC analysis of the two types of networks showed for both materials a weak endothermic transition between 50 and $60{ }^{\circ} \mathrm{C}$, which proved to be either melting of a crystalline anthracene dimer phase (A) or a liquid crystalline phase (B), based on evidence obtained by XRD measurements, which gave sharp reflections for $\mathbf{A}$ and a single broad reflection for $\mathbf{B}$. Polarization microscopy on $\mathbf{B}$ confirmed the presence of birefringent domains, typical for a nematic liquid crystalline phase. Above 125 and $60^{\circ} \mathrm{C}$, A and B behave like cross-linked rubbers up to $160-170{ }^{\circ} \mathrm{C}$, the onset of dimer dissociation. Above this point, both materials quickly lose their rubber characteristics and start to behave like viscoelastic liquids. In DSC, thermal dissociation of $\mathbf{3}$ is only slightly visible and was not visible for the network materials ( $\mathbf{A}$ and $\mathbf{B}$ ). This finding suggests that the splitting of dimer does not involve a significant change in enthalpy under these specific DSC conditions. Thermally dissociated anthracene dimer networks can be readily restored to a state that resembles their original state by irradiation with UVA, provided that the samples are homogeneous and no vitrification takes place during dimerization. In this particular case, for both networks $\mathbf{A}$ and $\mathbf{B}$, a slight heating to $60{ }^{\circ} \mathrm{C}$ was needed to prevent vitrification during the dimerization (A) or to create a homogeneous nonscattering sample (B).

\section{EXPERIMENTAL SECTION}

Materials and Instruments. Nuclear magnetic resonance spectra were recorded on a Bruker Avance $300(300 \mathrm{MHz})$, or a Bruker DRX $500(500 \mathrm{MHz})$ NMR spectrometer at room temperature. DSC experiments were performed on a TA Instruments Q2000 differential scanning calorimeter, equipped with either a refrigerated cooling system (RCS) or a liquid nitrogen cooling system (LNCS). A Tzero calibration was performed at $10 \mathrm{~K} \mathrm{~min}^{-1}$ using sapphire disks and temperature, and enthalpy calibrations were performed using indium calibration standards for Tzero hermetic aluminum pans. The instrument was purged using a $25 \mathrm{~mL} \mathrm{~min}{ }^{-1}$ nitrogen gas purge flow. Dynamic mechanical analysis (DMA) experiments were performed on a TA Instruments Q800 dynamic mechanical analyzer, equipped with a liquid nitrogen cooling system. All experiments are performed in tension using a film tension setup (TA Instruments), using rectangular specimens with a width of $2 \mathrm{~mm}$ and a thickness of $\sim 1 \mathrm{~mm}$. Dynamic rheometry experiments were performed on a TA Instruments AR-G2 dynamic rheometer equipped with an electrically heated plates setup using disposable aluminum parallel plates. For experiments under UVA irradiation, the lower plate assembly was replaced by a dedicated UV light guide accessory equipped with a disposable acrylic plate and connected to a Lumen Dynamics $100 \mathrm{~W}$ mercury arc lamp with $320-500 \mathrm{~nm}$ broad band gap filter $+1 \%$ neutral-density 2.0 filters. Measurements were performed in a $25 \mathrm{~mm}$ parallel plate setup using $200 \mu \mathrm{m}$ thick films. A Mettler-Toledo TGA/ SDTA 851e under nitrogen atmosphere was used for thermal dissociation of bulk dimers.

All chemicals were purchased from either ABCR, Sigma-Aldrich, TCI Europe, or Acros Organics and were used as such. IR spectra were recorded with a PerkinElmer FTIR SPECTRUM 1000 and a PIKE Miracle ATR unit. LC-MS analyses were performed on an Agilent Technologies 1100 series LC/MSD system with a diode array detector (DAD) and single quad MS. Analytical reversed phase HPLC-analyses were performed with a Phenomenex Luna C18 (2) column ( $5 \mu \mathrm{m}, 250$ $\mathrm{mm} \times 4.6 \mathrm{~mm})$ and a solvent gradient $\left(0-100 \%\right.$ acetonitrile in $\mathrm{H}_{2} \mathrm{O}$ in $15 \mathrm{~min}$ ), the eluted compounds were analyzed via UV detection (214 $\mathrm{nm})$. UV dimerization occurred in a Metalight Classic from Primotec, with 12 double $365 \mathrm{~nm}$ UV lamps of $9 \mathrm{~W}$ each.

Synthesis. 1,2,4-Tris(1-mercaptoethyl)cyclohexane, Mixture of (Stereo) Isomers (6). 1,2,4-Trivinylcyclohexane (mixture of isomers, $100 \mathrm{~mL}, 0.52 \mathrm{~mol}$ ) was cooled to $-10{ }^{\circ} \mathrm{C}$ in an ice--salt bath in a $1 \mathrm{~L}$ erlemeyer flask. Under vigorous stirring and continued cooling in the ice-salt bath, thioacetic acid $(121.5 \mathrm{~mL}, 1.55 \mathrm{~mol})$ was slowly added, followed by 2,2-dimethoxy-2-phenylacetophenone (1.5 g, $3.9 \mathrm{mmol}$ ). An efficient reflux cooler was then fitted, followed by the removal of the ice-salt cooling bath. After the initial exotherm had subsided, the reaction mixture was brought under argon and was irradiated for $1 \mathrm{~h}$, using a Metalight irradiation chamber, fitted with $365 \mathrm{~nm}$ fluorescent light bulbs. Subsequently, hydrochloric acid $(2 \mathrm{M}, 200 \mathrm{~mL})$ was added and the reaction mixture was then refluxed overnight. Next, the mixture was extracted with diethyl ether $(2 \times 200 \mathrm{~mL})$. The combined organic layers were then washed with brine $(2 \times 200 \mathrm{~mL})$, dried over magnesium sulfate and concentrated in vacuo, yielding a slightly yellow oil that was further purified by vacuum distillation $(0.1 \mathrm{mmHg} 130$ ${ }^{\circ} \mathrm{C}$ ) to yield 1,2,4-tris (2-mercaptoethyl)cyclohexane as a mixture of (stereo) isomers (6) (116 g, 85\%).

\section{ASSOCIATED CONTENT}

\section{Supporting Information}

The Supporting Information is available free of charge on the ACS Publications website at DOI: 10.1021/acs.macromol.6b02400.

NMR spectra of trithioacetate mixture (5) and trithiol mixture (6), GC-MS of 6, WAXRD of networks A and 
$\mathrm{B}$, and photocuring of thermally degraded $\mathrm{B}$ at $20{ }^{\circ} \mathrm{C}$ (PDF)

\section{AUTHOR INFORMATION}

Corresponding Author

*(F.D.P.) E-mail: filip.duprez@ugent.be.

ORCID $\odot$

Filip Du Prez: 0000-0003-4065-6373

\section{Author Contributions}

${ }^{\S}$ These authors contributed equally. The manuscript was written through contributions of all authors. All authors have given approval to the final version of the manuscript.

\section{Notes}

The authors declare no competing financial interest.

\section{ACKNOWLEDGMENTS}

This work was supported by FWO-Vlaanderen (Project G006913N). J.V.D. acknowledges Flanders Innovation \& Entrepreneurship for financial support. F.D.P. acknowledges the Belgian Program on Interuniversity Attraction Poles initiated by the Belgian State, the Prime Minister's Office (P7/05).

\section{REFERENCES}

(1) Kloxin, C. J.; Bowman, C. N. Covalent adaptable networks: smart, reconfigurable and responsive network systems. Chem. Soc. Rev. 2013, 42 (17), 7161-7173.

(2) Montarnal, D.; Capelot, M.; Tournilhac, F.; Leibler, L. Silica-Like Malleable Materials from Permanent Organic Networks. Science 2011, 334 (6058), 965-968.

(3) Denissen, W.; Rivero, G.; Nicolay, R.; Leibler, L.; Winne, J. M.; $\mathrm{Du}$ Prez, F. E. Vinylogous Urethane Vitrimers. Adv. Funct. Mater. 2015, 25 (16), 2451-2457.

(4) Denissen, W.; Winne, J. M.; Du Prez, F. E. Vitrimers: permanent organic networks with glass-like fluidity. Chem. Sci. 2016, 7 (1), 3038.

(5) Hyder Ali, A.; Srinivasan, K. S. V. Photoresponsive functionalized vinyl cinnamate polymers: Synthesis and characterization. Polym. Int. 1997, 43 (4), 310-316.

(6) Oya, N.; Sukarsaatmadja, P.; Ishida, K.; Yoshie, N. Photoinduced mendable network polymer from poly(butylene adipate) endfunctionalized with cinnamoyl groups. Polym. J. 2012, 44 (7), 724729.

(7) Nakayama, Y.; Matsuda, T. Photocycloaddition-induced preparation of nanostructured, cyclic polymers using biscinnamated or biscoumarinated oligo(ethylene glycol)s. J. Polym. Sci., Part A: Polym. Chem. 2005, 43 (15), 3324-3336.

(8) Xu, J. F.; Chen, Y. Z.; Wu, L. Z.; Tung, C. H.; Yang, Q. Z. Dynamic Covalent Bond Based on Reversible Photo $4+4$ Cycloaddition of Anthracene for Construction of Double-Dynamic Polymers. Org. Lett. 2013, 15 (24), 6148-6151.

(9) Van Damme, J.; Vlaminck, L.; Van Assche, G.; Van Mele, B.; van den Berg, O.; Du Prez, F. Synthesis and evaluation of 9-substituted anthracenes with potential in reversible polymer systems. Tetrahedron 2016, 72 (29), 4303-4311.

(10) Bouas-Laurent, H.; Castellan, A.; Desvergne, J.-P.; Lapouyade, R. Photodimerization of anthracenes in fluid solutions: (part 2) mechanistic aspects of the photocycloaddition and of the photochemical and thermal cleavage. Chem. Soc. Rev. 2001, 30 (4), 248-263.

(11) Manhart, J.; Ayalur-Karunakaran, S.; Radl, S.; Oesterreicher, A.; Moser, A.; Ganser, C.; Teichert, C.; Pinter, G.; Kern, W.; Griesser, T.; Schlögl, S. Design and application of photo-reversible elastomer networks by using the $[4 \pi \mathrm{s}+4 \pi \mathrm{s}]$ cycloaddition reaction of pendant anthracene groups. Polymer 2016, 102, 10-20.
(12) Hoyle, C. E.; Bowman, C. N. Thiol-Ene Click Chemistry. Angew. Chem., Int. Ed. 2010, 49 (9), 1540-1573.

(13) Hoyle, C. E.; Lee, T. Y.; Roper, T. Thiol-enes: Chemistry of the past with promise for the future. J. Polym. Sci., Part A: Polym. Chem. 2004, 42 (21), 5301-5338.

(14) Lowe, A. B. Thiol-ene "click" reactions and recent applications in polymer and materials synthesis. Polym. Chem. 2010, 1 (1), 17-36.

(15) Bowman, C.; Cramer, N.; Shandas, R.; Nair, D. P. Thiol-vinyl and thiol-yne shape memory polymers for medical use. WO2009132070A2, 2009.

(16) Hoyle, C. E.; Nazarenko, S.; Wei, H. Photocurable thiol-ene copolymer low gas permeability membranes. US20090253805A1, 2009.

(17) Woods, J. G.; Angus, R. O., Jr.; Schall, J. D. Thiol-ene cured oilresistant polyacrylate sealants for in-place gasketing applications. WO2009137197A2, 2009.

(18) Hawker, C. J.; Campos, L. M.; Meinel, I. Thiol-ene-based poly(alkylsiloxane) materials for lithography. US20090096136A1, 2009.

(19) Campos, L. M.; Truong, T. T.; Shim, D. E.; Dimitriou, M. D.; Shir, D.; Meinel, I.; Gerbec, J. A.; Hahn, H. T.; Rogers, J. A.; Hawker, C. J. Applications of Photocurable PMMS Thiol-Ene Stamps in Soft Lithography. Chem. Mater. 2009, 21 (21), 5319-5326.

(20) Bowman, C.; Anseth, K.; Hacioglu, B.; Nuttelman, C. Degradable thiol-ene polymers. US7288608B2, 2007.

(21) Bowman, C.; Lu, H.; Stansbury, J. Novel photopolymers and use in dental restorative materials. US20070082966A1, 2007.

(22) Bowman, C. N.; Cramer, N. B. Photopolymerizable resin systems for dental restorative materials. US20090270528A1, 2009.

(23) Rheinberger, V.; Moszner, N.; Salz, U. Polymerized alkoxysilyl derivatives of norbornene and mercaptans as dental materials. DE19619046A1, 1997.

(24) Coates, D.; Nolan, P.; Marden, S. A. Polymer-dispersed liquidcrystal system based on thiolene type polymer matrix precursor. GB2277744A, 1994.

(25) Chen, K.; Huang, H. Polymer electrolyte composition of saltcontaining thiol-ene matrix. WO9635238A1, 1996.

(26) Jacobine, A. F., In Radiation Curing in Polymer Science and Technology III, Fouassier, J. P., Rabek, J. F., Eds. Elsevier: London, 1993; Vol. III.

(27) Carlborg, C. F.; Haraldsson, T.; Oberg, K.; Malkoch, M.; van der Wijngaart, W. Beyond PDMS: off-stoichiometry thiol-ene (OSTE) based soft lithography for rapid prototyping of microfluidic devices. Lab Chip 2011, 11 (18), 3136-3147.

(28) Zhang, J.; Chen, Y.; Brook, M. A. Facile Functionalization of PDMS Elastomer Surfaces Using Thiol-Ene Click Chemistry. Langmuir 2013, 29 (40), 12432-12442.

(29) van den Berg, O.; Nguyen, L. T. T.; Teixeira, R. F. A.; Goethals, F.; Ozdilek, C.; Berghmans, S.; Du Prez, F. E. Low Modulus Dry Silicone-Gel Materials by Photoinduced Thiol-Ene Chemistry. Macromolecules 2014, 47 (4), 1292-1300.

(30) Payamyar, P.; Kaja, K.; Ruiz-Vargas, C.; Stemmer, A.; Murray, D. J.; Johnson, C. J.; King, B. T.; Schiffmann, F.; VandeVondele, J.; Renn, A.; Gotzinger, S.; Ceroni, P.; Schutz, A.; Lee, L. T.; Zheng, Z. K.; Sakamoto, J.; Schluter, A. D. Synthesis of a Covalent Monolayer Sheet by Photochemical Anthracene Dimerization at the Air/Water Interface and its Mechanical Characterization by AFM Indentation. Adv. Mater. 2014, 26 (13), 2052-2058.

(31) Goldbach, J. T.; Russell, T. P.; Penelle, J. Synthesis and thin film characterization of poly(styrene-block-methyl methacrylate) containing an anthracene dimer photocleavable junction point. Macromolecules 2002, 35 (11), 4271-4276.

(32) Song, Y. K.; Lee, K. H.; Hong, W. S.; Cho, S. Y.; Yu, H. C.; Chung, C. M. Fluorescence sensing of microcracks based on cycloreversion of a dimeric anthracene moiety. J. Mater. Chem. 2012, 22 (4), 1380-1386.

(33) Liu, B.; Wang, H.; Zhang, L.; Yang, G.; Liu, X.; Kim, I. A facile approach for the synthesis of cyclic poly(N-isopropylacrylamide) 
based on an anthracene-thiol click reaction. Polym. Chem. 2013, 4,

$2428-2431$.

(34) Fritz, D.; Walter, F.; Walter, S. Process for the production of 1,

2, 4-trivinylcyclohexane. US2967895A, 1961. 